\title{
THE ACCURACY OF HIGH S/N SPECTROSCOPIC MEASUREMENTS
}

\author{
Douglas K. Duncan \\ Space Telescope Science Institute \\ 3700 San Martin Drive \\ Baltimore, Maryland 21218 USA
}

\begin{abstract}
A careful intercomparison is made of several sets of high $\mathrm{S} / \mathrm{N}$ spectrsocopic observations made by different astronomers, using different telescopes and detectors, observing the same objects. Spectra of moderately weak (10-50 $\mathrm{mA}$ ) $\mathrm{Li}$ lines, observed at $\mathrm{S} / \mathrm{N}$ of one to several hundred using the CFHT Reticon, Lick Observatory Reticon, McDonald Observatory Digicon, and the Intermediate Dispersion Spectrograph plus CCD of the Isaac Newton Telescope agree within the claimed measurement accuracies of about 3-6 mA almost without exception. CFHT Reticon observations of Hyades dwarfs are compared with unpublished observations taken with a CCD at Lick Observatory; again, the agreement is very good. However, abundance determinations based on these measurements agree less well. The discrepancy arises from different temperatures used by different investigators for the same stars. The accuracy of various methods of temperature determination is examined, including $B-V, R-I$, and V-K colors, continuum scans, $\mathrm{H} \alpha$ line profile fitting, and temperatures derived from fine analyses. With careful work and good data, temperatures still limit the accuracy of some of the programs discussed.
\end{abstract}

\section{INTRODUCTION}

The development of more sensitive and more linear detectors over the last several years has permitted spectra of higher $S / N$ to be obtained. New questions are being addressed, the answers to which depend on great accuracy in spectroscopic and abundance measurements. Measurements of slight abundance differences between stars or small changes in line profiles with time in a given star [e.g. Doppler Imaging] are examples. Especially when results obtained at different observatories are compared, one wants to know whether the quoted $\mathrm{S} / \mathrm{N}$ values are indicative of all the errors of measurement. More generally, are abundance determinations based on high $\mathrm{S} / \mathrm{N}$ data achieving the accuracy claimed?

A recent investigation, done with L. Hobbs (Hobbs and Duncan, 1987), required a very careful intercomparison of abundances obtained at different observatories, with different equipment. The investigation was of the abundance of $\mathrm{Li}$ in halo stars. Standard models of the big bang (cf. the review of Boesgaard and Steigman, 1985) predict the formation of $\mathrm{He}, \mathrm{D}$, and ${ }^{7} \mathrm{Li}$, in amounts which are a strong function of the baryon to photon ratio. Measurements of the primordial 
abundance of any of these isotopes thus have important cosmological significance.

$\mathrm{Li}$ is a trace element. In a wide variety of pop. I objects it is found with a logarithmic abundance of $n_{L i} \approx 3.0$ (where $n_{H}=12.00$ ). However, solar type stars gradually destroy $\mathrm{Li}$, and the current abundance in the sun is only $\mathrm{n}_{\mathrm{Li}}$ $\approx 1.0,100 \mathrm{X}$ less than that with which it formed. It might be expected that halo stars, much older than the sun, would have destroyed essentially all their $\mathrm{Li}$. Thus it was an important and surprising discovery when Spite and Spite (1982), and Spite, Maillard, and Spite (1984) showed that all the extremely metal-poor stars of temperature $5600 \mathrm{~K}$ to $6300 \mathrm{~K}$ they observed showed $\mathrm{Li}$ abundances of $\mathrm{n}_{L i}=2.05 \pm 0.2$. Apparently the lack of metals in these stars leads to thinner convection zones which do not mix $\mathrm{Li}$ to regions which destroy it as fast as in the sun.

The $\mathrm{Li}$ abundance observed in the halo stars is 10X less than is seen in pop. I objects, and this naturally raises the following question. Did the big bang produce an abundance $n_{L i} \approx 2$, and galactic sources (presently unknown) raise the abundance to the currently observed value $n_{L i} \approx 3$, or did the big bang produce $n_{L i} \approx 3$, and the halo stars deplete their $\mathrm{Li}$ about one order of magnitude, but not the two orders of magnitude seen in the sun. If the halo stars all truly have the same abundance, the former hypothesis is prefered, but if small star-to star differences exist it is an indication that at least some destruction has occurred, even in the halo stars.

\section{A COMPARISON OF DIFFERENT LI ABUNDANCE DETERMINATIONS IN HALO STARS}

\subsection{Equivalent width (EW) comparisons}

Hobbs and Duncan, first working independently and later together, obtained data on many additional halo stars and compared their results to those of Spite et al. The Duncan-Hobbs spectra were obtained mainly with a Lick Observatory coude camera and bare Reticon detector, and a McDonald Observatory coude camera and Digicon detector. The former has a resolution of $0.3 \hat{A}$ and the latter $0.26 \hat{A}$. Signal to noise was typically 80 , and the claimed accuracy 3-7 $\mathrm{m} A$, which represents $10-20 \%$ in these moderately weak lines. Five stars were observed both at Lick and McDonald. Their measured EWs showed very good agreement, with a mean difference of $0 \pm 4 \mathrm{~mA}$. Twelve stars already observed by Spite et al. were repeated in order to permit a detailed intercomparison of results.

The Spite et al. spectra were obtained at the coude focus of the Canada France Hawaii Telescope (CFHT). The detector was a bare Reticon, the resolution $0.26 \lambda$, and typical $S / N$ 100. Figure 1 compares the two sets of results. Agreement is seen to be very good, with rms. difference for the 12 stars $0 \pm 4$ mA.

\subsection{Abundance Determination}

Figure 2 shows the $\mathrm{Li}$ abundances determined by Spite et al. vs. the DuncanHobbs values. Although the mean difference is close to zero, the scatter about the diagonal line of perfect agreement is large, std. dev. $\sigma=0.15$. An EW error of $20 \%$ in these lines corresponds to an abundance error of 0.08 dex; this cannot be the main source of scatter. 


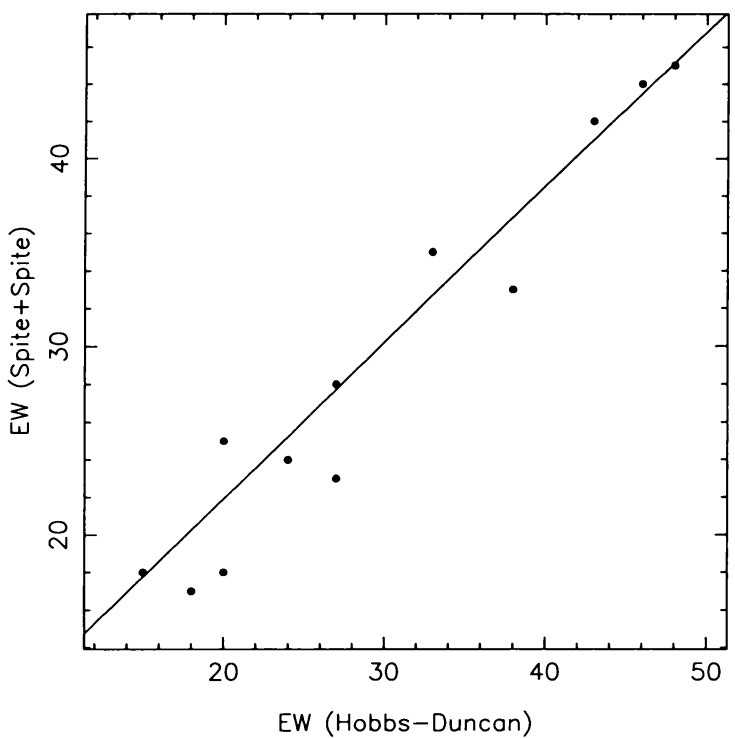

Figure 1. An equivalent width comparison shows very good agreement.

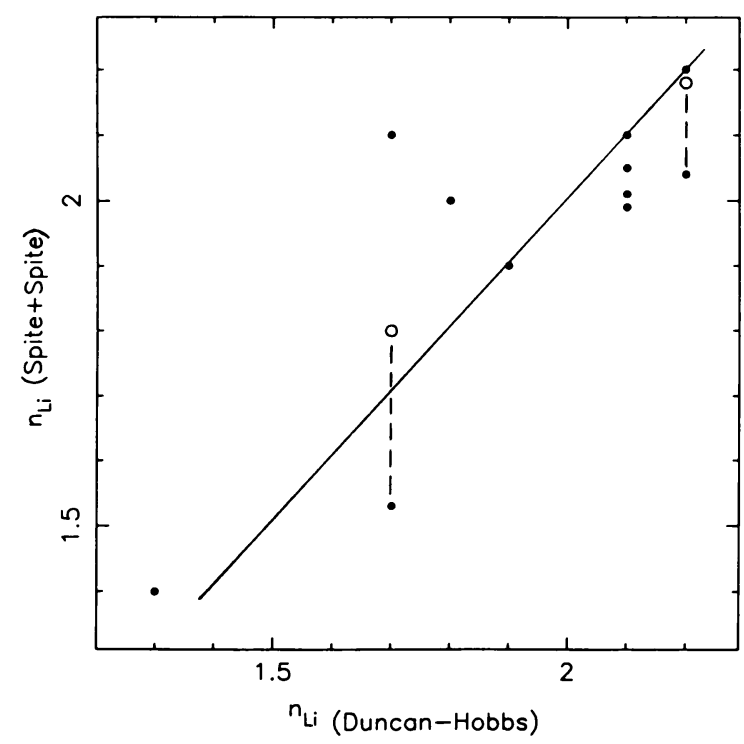

Figure 2. Comparison of $\mathrm{Li}$ abundance determinations. Two open circles show the effect of correcting temperatures as explained in the text.

\subsection{Temperature Comparison}

Figure 3 makes a comparison of effective temperatures used for the same stars 
in the two separate investigations. The scatter is relatively large, with an rms. difference of $120 \mathrm{~K}$. This corresponds to an abundance change of about $0.12 \mathrm{dex}$, and explains most of the scatter in Figure 2.

Temperatures in the Spite et al. investigations were determined from fine analyses and R-I colors. In an effort to get the most uniform possible temperatures, Hobbs and Duncan considered R-I and V-K colors, continuum scans made by Peterson and Carney (1979), and Stromgren colors. In two cases, reasons were found to doubt the temperatures taken from the literature by the Spites. If those two stars are re-analyzed with new temperatures, their abundances change as shown by the open circles in Figure 2. Hobbs and Duncan found that temperatures determined from fine analyses were the least consistent of all sources examined.

Comparing different sources of temperatures for all their halo stars, Hobbs and Duncan found an rms. difference of $55 \mathrm{~K}$ between $\mathrm{T}$ (continuum scan) and $T(V-K)$, and an rms. difference of $80 \mathrm{~K}$ between $\mathrm{T}(\mathrm{scan})$ and $\mathrm{T}(\mathrm{R}-\mathrm{I})$. Random errors, which are more important than the zero-point calibration of the temperature scale when one is interested in differential abundance comparisons between stars, should be reduced by averaging independent temperature determinations. Hobbs and Duncan conclude that their relative temperature errors are $60 \mathrm{~K}$. This compares with the accuracy estimated by Peterson and Carney themselves, of $80 \mathrm{~K}$, from combining R-I, V-K, and scan temperatures.

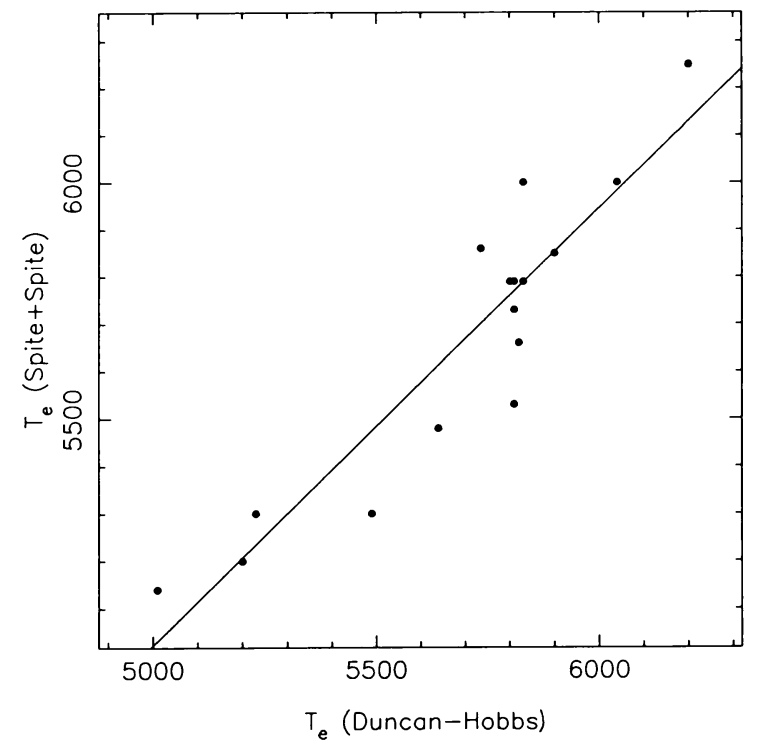

Figure 3. Comparison of temperatures used for the same stars in two separate investigations.

\subsection{Another independent comparison}

Quite recently Rebolo, Molaro, and Beckman (RMB, 1987) have finished a very comprehensive study of $\mathrm{Li}$ abundances in halo dwarfs. Their conclusions agree 
with those of Hobbs and Duncan and those presented here, and, for six stars which are in common, allow another external check of measurement accuracy. The RMB observations were made at the Cassegrain focus of the Isaac Newton telescope at the Observatorio del Roque de los Muchachos (La Palma). The intermediate dispersion spectrograph plus CCD yielded a resolution of about $0.44 \lambda$. Typically $S / N$ of about 150 was achieved, leading to an estimated measurement accuracy of $10 \%$ in EW. Comparison of the RMB results with those of Hobbs and Duncan shows a mean EW difference of $0 \pm 4 \mathrm{~mA}$, in very good accord with the accuracy claimed by each set of authors. Since the RMB determinations of temperature are based on some of the same sources used by Hobbs and Duncan, they do not provide independent information.

\section{ARE THE STAR-TO-STAR DIFFERENCES IN HALO LI ABUNDANCE REAL?}

With the best-determined temperatures and equivalent widths in hand, we now confront the question whether the star-to-star abundance differences real. We focus attention on 6 very metal-poor stars of almost exactly the same temperature, listed in Table I.

\section{TABLE I}

\begin{tabular}{|r|c|c|c|c|c|c|c|}
\hline HD or BD & R-I & V-K & T $_{\text {scan }}$ & $\mathrm{EW}_{D H}$ & $\mathrm{EW}_{S S}$ & $\mathrm{EW}_{R M B}$ & $\mathrm{n}_{L i}$ \\
\hline 19445 & .345 & 1.39 & 5820 & 38 & 33 & 35 & 2.1 \\
94028 & .33 & 1.39 & - & 33 & 35 & & 2.1 \\
201891 & .33 & 1.42 & 5810 & 27 & 23 & 27 & 1.9 \\
219617 & .33 & 1.41 & 5800 & 43 & 42 & 40 & 2.2 \\
+174708 & .34 & 1.40 & 5810 & 25 & & 25 & 1.9 \\
+263578 & .33 & & & 24 & 24 & & 1.8 \\
\hline
\end{tabular}

Contributions to the expected error in $\mathrm{Li}$ abundance determination include 0.08 dex, if the EW errors are $20 \%$ (they appear to be somewhat better here), and 0.08 dex from an effective temperature uncertainty of $80 \mathrm{~K}$, for a combined error of 0.11 dex. The observed rms. scatter about the mean is 0.15 dex. This leads to a formal reduced chi-square value of 2.1 , with a $5 \%$ probability of scatter as large as observed being due to chance. The point farthest from the mean is HD 219617, and if this star was not included in the analysis, the scatter would be 0.134 , with a probability $20 \%$ of arising from chance. HD 219617 is a binary with nearly identical components, but there is no particular reason to think that this would effect its $\mathrm{Li}$ abundance. In the end, we must conclude as did Hobbs and Duncan: there is marginal, but not compelling evidence of real differences.

RMB make an important arguement concerning $\mathrm{Li}$ destruction. They point out that halo stars somewhat cooler than those of Table I, which do show $\mathrm{Li}$ destruction, actually have destroyed their $\mathrm{Li}$ much more slowly than pop. I stars of the same temperature. As one moves to higher temperatures in pop. I stars, the rate of $\mathrm{Li}$ destruction decreases rapidly (cf. the Hyades observations of Cayrel et. al 1984). If the same pattern obtains in pop. II, the stars in Table I could not have altered their $\mathrm{Li}$ abundances from that with which they formed. 


\section{POPULATION I OBJECTS}

Equivalent width determinations are simplified in metal-poor objects by the smoothness of the continuum. Aadditional comparison was therefore made between two independent sets of abundance determinations in the same group of pop. I objects. These were $\mathrm{Li}$ abundance determinations in Hyades dwarfs. The first observations were those of Cayrel, Cayrel, and Cambell $\left(C^{3}, 1985\right)$, who obtained spectra of $S / N \approx 250$ and resolution $0.2 A$ with the CFHT Reticon. The others were unpublished observations made by $D$. Soderblom with a coude camera and CCD detector at Lick Observatory, at somewhat lower S/N. No systematic differences in EW were detected. Cayrel et al. derived temperatures from fitting $\mathrm{H} \alpha$ profiles, and they claim a random error of only $30 \mathrm{~K}$ in this method. Comparing their $\mathrm{H} \alpha$ temperatures with ones derived from B-V, R-I, and V-K colors (slightly adjusting scale zero points to match) results in scatter of $70 \mathrm{~K}, 70 \mathrm{~K}$, and $40 \mathrm{~K}$ respectively. Again accurate temperatures are critical to the abundance determination.

\section{CONCLUSION}

Equivalent widths determined at high $\mathrm{S} / \mathrm{N}$ with a number of different telescopes and spectrographs show every indication of being as accurate as the authors claim. All of the examined spectra were in the red part of the spectrum in solar type stars, or in metal-poor stars, where the continuum is relatively uncluttered. Abundances determined from the spectra often agree less well, due to different temperatures used in the analyses. These results emphasize the need for stellar parameters of accuracy commensurate with the high accuracy of the spectra themselves, to be used for input in abundance analyses. In some cases it may be advantageous to derive these directly from the spectra, e.g. temperatures from balmer-line profiles.

In the interesting case of the $\mathrm{Li}$ abundance in very metal-poor dwarfs, the most careful investigations produce no conclusive evidence that the $\mathrm{Li}$ observed is anything other than the uniform primordial abundance produced by the big bang.

I would like to than R. Rebolo and D. Soderblom for communicating work in advance of publication.

\section{REFERENCES}

Boesgaard, A.M., and Steigman, G. 1985, Ann. Rev. Astr. Ap., 23, 319.

Cayrel, R., Cayrel de Stroebel, G., Campbell, B., and Dappen, W. 1984, Ap. J., 283, 205.

Cayrel, R., Cayrel de Stroebel, G., and Campbell, B., 1985, Astr. Ap., 146, 249.

Hobbs, L.M. and Duncan, D.K. 1987, Ap. J., s17, 796.

Peterson, R.C., and Carney, B.W., 1979, Ap. J., 231, 762.

Rebolo, R., Moloro, P., and Beckman, J.E. 1987, preprint.

Soderblom, D., 1987, private communication.

Spite, F., and Spite, M. 1982, Astr. Ap., 115, 357.

Spite, M., Maillard, J.P., and Spite, F. 1984, Astr. Ap., 141, 56. 


\section{DISCUSSION}

PEILRSON All the stars hot enough show the same lithium abundance, although their iron abundances range from $-3.0<[\mathrm{Pe} / \mathrm{B}]<-1.4$ does this imply, in your opinion, that the lithium abundance is cosmological?

DUNCAN I think it suggests it, yes.

BOSSGAARD Yes, lithium is decoupled from the Fe abundance in halo dwarfs. However, Li diffusion (as opposed to depletion by nuclear burning) could occur in a way that is not dependent on the metallicity during the 15 billion year lifetimes of these stars. I think the halo star Li content should be interpreted as a lower limit to the Big Bang Li production.

DUnCAN one cannot preclude this, and a conservative position is that the Li abundance is a lower limit. However, there is no reason to suppose this, either. I know of no theoretical calculations which predict Ii depletion in Pop.II stars, independent of temperature. If I become convinced that star to star differencies are real, then I will believe in depletion, that is the best evidence.

MOLARO you said that, may be, there is a scatter in the Ii abundances on the "plateau". Assuming this as real, does it show some trend with temperature?

DUNCAN The scatter shows no trends with Te or $[\mathrm{Fe} / \mathrm{H}]$, as long as $[\mathrm{Fe} / \mathrm{H}]<-1.4$.

SODERBLOM Ann Boesgaard suggested that diffusion, for example, could influence the apparent $\mathrm{Li}$ abundance of the Pop.II stars. Can anyone suggest tests to tell if a process like that has occured ? (no answer...) 